models. A sensitivity analysis was carried out stratifying by sport PA levels.

Result In a median 17 years of follow-up, $n=102$ first CVD events occurred. As compared to the intermediate OPA tertile, workers with low and high OPA showed higher HRs of 1.67 (95\% CI: 0.96 to 2.92 ) and 2.01 (1.17-3.46), respectively. Stratifying by sport PA, the above reported HRs for low and high OPA workers increased to $2.32(1.15 ; 4.69)$ and to 2.54 $(1.09 ; 5.95)$ when sport PA was below and above the median, respectively. High vs non-high JS workers evidenced an HR of 1.27 (0.76-2.11). When adjusting for age, BMI, alcohol intake, smoking and sport PA, a joint effect was detected between OPA and JS, with the highest HR for workers in the low OPA and high JS category $[2.70(1.17 ; 6.26)]$ as compared to workers in the intermediate OPA and non-high JS.

Discussion We observed a joint additive effect between sedentary work and high JS on the incidence of cardiovascular events.

\section{C CARDIOVASCULAR DISEASE SCREENING AT THE WORKPLACE: DISCRIMINATION ABILITY OF LIFESTYLE RISK FACTORS AND JOB-RELATED CONDITIONS}

${ }^{1} \mathrm{G}$ Veronesi ${ }^{*},{ }^{1,2} \mathrm{~F}$ Gianfagna, ${ }^{3} \mathrm{R}$ Borchini, ${ }^{4} \mathrm{G}$ Grassi, ${ }^{1,2} \mathrm{~L}$ lacoviello, ${ }^{4} \mathrm{G}$ Cesana, ${ }^{1} \mathrm{P}$ Tayoun, ${ }^{1,3} \mathrm{MM}$ Ferrario. 'Research Centre in Epidemiology and Preventive Medicine, Department of Medicine and Surgery, University of Insubria, Varese, Italy; ${ }^{2}$ IRCCS Neuromed, Pozzilli, Italy; ${ }^{3}$ Occupational Medicine Unit, Varese Hospital and University of Insubria, Varese, Italy; ${ }^{4}$ Department of Medicine, University of Milano-Bicocca, Monza, Italy

\subsection{6/oemed-2018-ICOHabstracts. 172}

Introduction Lifestyle and job-related conditions (LS and JRC) are recognised cardiovascular disease (CVD) risk factors, but their prognostic utility remains to be established. We investigated the discrimination ability at 10 years of LS and JRC in a Northern Italian working male population.

Methods $n=2532$ men, 35-64 years, CVD-free and employed at the time of recruitment (1989-1996) in either the MONICA-Brianza and PAMELA (population-based) or the SEMM (factory-based) cohorts, were available for the analyses. The following LS and JRC were ascertained using standardised procedures: smoking (current vs non-current); alcohol intake (drinks/day; 1-3 drinks as reference); habitual occupational and sport physical activity (PA; the Baecke questionnaire); job strain (high vs non-high; Job Content Questionnaire) and BMI, from measured height and weight. Workers were followed-up (median 14 years) until first major coronary event or ischaemic stroke, fatal or non-fatal. Discrimination ability was estimated as the Area Under the ROC-Curve (AUC) for a Cox model with LS and JRC satisfying the Akaike Information Criterion for the selection of candidate predictors, and contrasted to the AUC from a model including blood lipids, blood pressure, smoking and diabetes.

Result $\mathrm{n}=162$ events occurred during follow-up (10 year risk: 4.3\%). BMI was not associated with the endpoint. The risk factors meeting the AI Criterion were: smoking (Hazard Ratio $=2.49$, 95\% CI: 1.81 to 3.42); alcohol intake (abstainers: $\mathrm{HR}=1.52,1.03-2.23 ; 6+$ drinks/day: $\mathrm{HR}=1.81,1.11-2.95)$; job strain $(\mathrm{HR}=1.39,0.98-1.97)$; combined sport and occupational PA $(p=0.02)$, as the HRs for sport PA changed between workers at low $(\mathrm{HR}=0.42)$ and intense $(\mathrm{HR}=1.55)$ occupational PA (interaction test $\mathrm{p}=0.001$ ). The LS and JRC model had the same discrimination (AUC $=0.75 ; 95 \% \mathrm{CI}$ : 0.70 to 0.78 ) than the model with clinical and biological risk factors
$(\mathrm{AUC}=0.75)$; this finding was consistent across the occupational classes.

Discussion Our results support the potential of primary prevention interventions at the workplace based on promoting healthier lifestyles and on improving job-related risk factors, to reduce CVD risk among workers.

\section{1d PSYCHOSOCIAL STRESS AT WORK INCLUDING JOB INSECURITY - IMPORTANT RISK FACTORS OF CHD: AN UPDATED SYSTEMATIC REVIEW}

1J Siegrist*, 2J Li. 'Senior Professorship, Faculty of Medicine, University of Düsseldorf, Germany; ${ }^{2}$ Institute of Occupational and Social Medicine, Faculty of Medicine, University of Düsseldorf, Germany

\subsection{6/oemed-2018-ICOHabstracts.173}

Introduction In recent years evidence on elevated risks of coronary heart disease (CHD) due to exposure to adverse, chronically stressful psychosocial working conditions has accumulated. Exhausting demands and efforts in combination with low control and low reward at work seem to be of particular significance, augmenting physiological dysregulation of the cardiovascular system. Two theoretical models, demand-control and effort-reward imbalance, assessing these conditions, have been studied most frequently in recent past. This contribution aims at providing a synthesis of the current state of art concerning cohort studies on associations of these models with CHD.

Methods We conducted a systematic review on published and unpublished prospective epidemiologic studies estimating the relative risk of CHD as a function of either model. As results are often given for effects of single model components in addition to their summary measures this information is additionally analysed. This holds particularly true for job insecurity as this component has often been studied as a single risk factor. Reviews were conducted according to established quality criteria (PRISMA).

Result Taken together, both models were associated with a significantly elevated relative risk of CHD. Consistency of findings was higher if single components were analysed, specifically in case of low job control and high job insecurity. Moreover, the strength of associations varied according to adjustment for confounding factors, and there was limited evidence of a moderating role of socioeconomic position.

Discussion This review found support for a role of chronic psychosocial stress at work in terms of two theoretical models, demand- control and effort-reward imbalance, as risk factors of CHD. While the risk elevation was modest, the prevalence of exposure to stressful conditions at work was high, thus emphasising the relevance of findings for occupational health. Future studies should combine these models and enlarge the scope of analysis. Moreover, intervention studies are needed to strengthen the evidence base.

\section{1e CARDIOVASCULAR AUTONOMIC ASSESSMENT FROM THE CLINICAL LABORATORY TO THE OCCUPATIONAL ENVIRONMENT. A NEW CHALLENGE TO PREVENT CARDIOVASCULAR DISEASES IN WORK PLACE}

F Barbic. Department of Internal Medicine, Humanitas Research Hospital, Humanitas University, Rozzano, Italy

10.1136/oemed-2018-ICOHabstracts.174 
Introduction Autonomic Nervous System allows a proper response to different environmental stimuli and is crucial for worker to adapt to physical effort and psychological stress i.e. shift-work, environmental temperature changes, long-lasting gravitational stimulus, physical demand.

Methods ECG, beat-by-beat Blood Pressure and Respiration are recorded while supine and during gravitational stimulus ( $75^{\circ}$ Head-up tilt). Sinus Arrhythmia and Valsalva Manoeuvre provide the relationship between respiratory activity and heart rate and the integrity of baroreceptors functioning. Hyperventilation, mental arithmetic, exposure to noise or occupational pollutants may also be used.

ECG and BP are recorded during working and sleeping time over 24 hours or more.

Spectral analysis provides indexes of cardiovascular autonomic control. From RR variability, Low Frequency $\left(\approx 0.1 \mathrm{~Hz}, \mathrm{LF}_{\mathrm{RR}}\right)$ and High Frequency $\left(\approx 0,25 \mathrm{~Hz}, \mathrm{HF}_{\mathrm{RR}}\right)$ oscillatory components are indexes of cardiac sympathetic and vagal modulation, respectively. LF/HF quantify the instantaneous cardiac sympatho-vagal tone. From systolic arterial pressure (SAP) variability, Low Frequency oscillatory component $\left(\approx 0.1 \mathrm{~Hz}, \mathrm{LF}_{\mathrm{SAP}}\right)$ is an index of vascular sympathetic modulation. Baroreflex sensitivity are assessed in the time (BRSseq) and frequency ( $\alpha L F)$ domain.

Symbolic analysis of heart period may furnish additional non-linear tools to quantify the sympatho-vagal modulation to the heart.

Results In susceptible workers, triggers involving autonomic nervous system, potentially present in the work environment (mental, visual, visceral or orthostatic stress) may promote neuro-mediated syncope or orthostatic intolerance syndromes.

Hypertensive workers show an early cardiovascular sympathetic predominance with 24 hour sympatho-vagal balance impairment

In healthy shift workers a blunted circadian oscillations of heart rate and $\mathrm{LF} / \mathrm{HF}$ during morning and night shift may represent a possible early sign of misadaptation to shift work in absence of any disease.

Discussion The assessment of individual cardiovascular autonomic profile in clinical laboratory and during working activity may help prevention of cardiovascular disease and promote health and safety at work by a 'Precise Medicine' approach.

\section{DEVELOPMENT OF EATING PATTERN FOR PEOPLE WITH UNCONTROLLED HYPERTENSION USING FAMILY PARTICIPATION APPROACH}

${ }^{1}$ B Duangduen*, ${ }^{2}$ A Sivaporn, ${ }^{2}$ B Waraporn. I'Instructor, Chiang Mai University, Chiang Mai, Thailand; ${ }^{2}$ Assistant Professor, Chiang Mai University, Chiang Mai, Thailand

\subsection{6/oemed-2018-ICOHabstracts. 175}

Introduction Eating is essential for controlling blood pressure level among hypertensive worker, therefore it is important that eating patterns should suit the context and lifestyle of these persons.

Methods A developmental research design was used to conduct this study. This study aimed to develop eating patterns for hypertensive worker with uncontrolled hypertension using family participation based on the family participation concept from related literature reviewed and to study the feasibility of using the eating pattern in real situations. The population in the study consisted of:

- hypertensive worker with uncontrolled hypertension $(\mathrm{N}=68)$,
- their family members $(\mathrm{N}=68)$, and

- Healthcare volunteer villages and Health promoting hospital staff responsible for chronic illness $(\mathrm{N}=11)$.

The study instruments were comprised of:

- People with Uncontrolled Hypertension Eating Questionnaire,

- Family Members Eating Questionnaire,

- Focus Group discussion guide for Village Healthcare Volunteers and Health promoting Hospital Staff,

- Feasibility of Using the Eating Pattern in Real Situation Questionnaire, and

- Developmental Plan of the eating pattern for people with uncontrolled hypertension using family participation approach.

The data were analysed using descriptive statistics and qualitative analysis.

Results Two main results were found. The data from situation analysis phase documented that the workers still had improper eating behaviour and their main obstacle for improving their eating behaviour is time and their socioeconomic status. Secondly, the new eating pattern were comprised of three main ideas: increasing consumption of vegetables, following a low salt diet criteria, and appropriate eating. And the result of possibility for using new eating pattern showed the new plan was suitable for the lifestyle of worker with the uncontrolled hypertension and their family members at a moderate level $(x=2.50$, S.D. $=0.62)$ and was feasible $(x=2.47$, S.D. $=0.61)$. In addition, the worker suggested that it is important to use mass media to provide more information about eating patterns to highlight the future benefits for uncontrolled hypertensive persons. Communities should encourage this group and their family members to use the eating plan and promote this eating pattern in the community and in the future, the eating plan should include recipes for hypertensive that use local vegetables known to lower high blood pressure. These recipes should be easy for to prepare.

Conclusion In conclusion, although this study could explore the eating situation of the worker with uncontrolled hypertension and can created the new eating plan, but for future study, we should to study more about how to improve the uncontrolled hypertension work eating behaviour following this finding.

\section{CARDIOVASCULAR REACTION TO STRESSFUL SITUATIONS IN PUBLIC TRANSPORT DRIVERS}

J Siedlecka*, E Gadzicka, T Makowiec-Dąbrowska, A Bortkiewicz. Nofer Institute of Occupational Medicine, Lodz, Poland

\subsection{6/oemed-2018-ICOHabstracts.176}

Introduction The job of a bus driver in public transport is characterised by an increased risk of cardiovascular diseases, including hypertension. The present project has been focused on investigating the cardiovascular response to professional duties and work-related stress in public transport drivers with treated and untreated arterial hypertension vs the drivers with normal blood pressure values.

Methods The study group were 61 drivers aged 37-58 years. Thirty of them had a hypertension: 15 subjects received systematic treatment and the other 15 had no hypotensive therapy. Normal BP values were found in 31 subjects. All the subjects had general medical examination, responded to a questionnaire 